\title{
Evolución y Desarrollo de los Modelos de Intervención Psicoeducativa en España
}

\section{Development and Evolution in Spain of Models of Psycho-educational Intervention}

\author{
Adolfo Fernández Barroso* \\ Director de Psicología Educativa y Presidente de la Sección de Psicología Educativa del Colegio Oficial de Psicólogos de Madrid
}

\begin{abstract}
Resumen. En el presente trabajo se plantean algunas reflexiones sobre la intervención del psicólogo en el Sistema Educativo. Para ello se revisan algunos de los factores que han condicionado el desarrollo de la Psicología Educativa aplicada en España, analizando sus orígenes y líneas de evolución, así como los aspectos de presencia profesional y rol. Asimismo se exponen las dificultades que se han presentado en dicho desarrollo y las posibles perspectivas de futuro de la intervención psicoeducativa paralelamente a las características de las actividades formativas que pueden garantizar una actuación profesional de calidad y al servicio de la comunidad social.

Palabras clave: Psicología de la Educación, Psicología Escolar, formación del psicólogo educativo, rol profesional, intervención psicoeducativa, formación profesionalizadora, sistema educativo.
\end{abstract}

\begin{abstract}
In this study, we present some reflections on interventions by psychologists in the Educational System. To this end, we review some factors that have conditioned the development of applied educational psychology in Spain, analyzing their origins and evolution, as well as aspects of professional presence and role. We also set out the difficulties that have arisen in the past, and the possible future development of psycho-educational intervention. At the same time, the types of training activities that can best ensure quality performance and social service community are also described.

Keywords: Educational Psychology, School Psychology, Educational psychologist training, professional role, psycho-educational intervention, professional training, education system.
\end{abstract}

\section{Introducción}

Inicialmente en este artículo trataremos de realizar una breve descripción de las diferentes circunstancias y cambios que han influido en la labor profesional de los psicólogos educativos del estado español en los últimos decenios (no entraremos en el análisis de los aspectos académicos, hacerlo requeriría una extensión y profundidad que sobrepasaría los

\footnotetext{
*Articulo póstumo (Conferencia impartida en el área de Psicología Educativa y del Desarrollo durante el III Congreso Iberoamericano de Psicología. 26 de julio de 2002. Bogotá, Colombia).
}

límites del presente trabajo y seguramente de su autor). A partir de esta descripción trataremos de extraer algunas referencias válidas para plantear posibles modelos formativos de carácter profesionalizador.

En el desarrollo histórico de la Psicología Educativa (Muñoz, García y Sánchez, 1997) podemos delimitar cuatro fases de acuerdo con la aparición de las funciones más significativas que la psicología educativa ha ido asumiendo. Así la primera época (1880-1920) se caracteriza por la preocupación por el estudio de las diferencias individuales y la admi- 
nistración de tests útiles para el diagnóstico y tratamiento de los niños problemáticos, de modo que en sus orígenes la psicología educativa aparece fuertemente ligada a la educación especial. En un segundo momento (1920-1955) el impacto del movimiento de salud mental promueve la proliferación de servicios psicológicos para tratar los problemas psicológicos infantiles dentro y fuera de la escuela y divulga la idea de una psicología "escolar" no limitada al diagnóstico y tratamiento de los problemas de aprendizaje escolar, sino ocupada también en la atención a los aspectos emocionales, afectivos y sociales del alumno.

En la tercera fase (1955-1970) empieza a considerarse la necesidad de formar a los profesores en los avances del conocimiento psicológico y en su integración en la metodología didáctica y se piensa en el psicólogo como el profesional que actúe de puente entre tal conocimiento psicológico y la práctica escolar. A partir de 1970, comienza la búsqueda de modelos alternativos basados en las teorías cognitivas, sistémicas, organizacionales, ecológicas y en la psicología comunitaria intentando dar un giro al esquema tradicional de atención individualizada a los casos problemáticos subrayando la importancia del contexto, tanto instruccional como sociocomunitario.

Con respecto a nuestro país los inicios de la psicología educativa están unidos a los comienzos de la psicología científica ya que fue el interés en la psicología aplicada al contexto escolar y a la orientación profesional el motor de desarrollo de esta disciplina. Hasta la guerra civil se producen diversas experiencias precursoras que se ven cortadas por dicho acontecimiento y por las características represivas y reaccionarias de la dictadura que la continúa.

A partir de los años cincuenta se produce una recuperación de la tradición científica que se concreta con la llegada de la Psicología, en la década de los sesenta, al mundo académico lo cual supone un salto cualitativo hacia su institucionalización pese a las fuertes tensiones estructurales que se dan en su seno (entre investigación y profesión, entre formación básica y especializada, así como por la diversidad teórica de partida debida en parte a la variabilidad de escuelas y enfoques con que cuenta la psicología). A este respecto pueden hacerse dos matizaciones. Por un lado, la explosión demográfica de titulados en psicología ha venido más de la demanda vocacional que de la demanda específica de tales especialistas en el mercado laboral, salvo quizás en el campo educativo donde ha habido un desarrollo significativo de la atención a los temas psicoeducativos. Por otro lado, existe un fuerte distanciamiento entre la dimensión académica y la profesional que repercute en la cualificación de especialistas en psicología aplicada.

A partir de los años setenta se configura una demanda social de intervención psicoeducativa. Esta demanda, asociada tanto a la difusión y generalización de ideas "psicologizantes" como a la extensión de la oferta de los primeros titulados, se concreta en las primeras prácticas de psicología educativa, centradas desde un enfoque psicotécnico en actuaciones de aplicación de pruebas, informes estandarizados, orientación en cursos claves y ocasionalmente en actividades de reeducación. Los protagonistas eran psicólogos que independientemente o en grupo ofrecen sus servicios y establecen relaciones laborales irregulares con asociaciones de padres de alumnos, propiedad y dirección de centros escolares privados, etc.

\section{La Psicología Educativa en la enseñaza privada}

Es en la enseñanza privada (concertada o propiamente privada) donde comienzan a trabajar las primeras promociones de psicólogos del área educativa. Desde esos inicios como servicios complementarios y extraescolares hasta el momento actual su evolución podría resumirse en:

A) Tendencia a la desaparición de la actuación exclusivamente psicotécnica y psicométrica, además de haberse consolidado la presencia de empresas que ofrecen servicios estadísticos y técnicos de calidad así como material psicológico que cubre esta faceta. 
B) Han aumentado los servicios derivados de la presencia continua del psicólogo en el centro escolar en una situación más regularizada de trabajo, aunque aún ahora, las condiciones laborales (estabilidad, horario, dedicación, etc.) sean muy diversas.

C) Si bien es cierto que la introducción del psicólogo en los centros fue acompañada inicialmente de una acentuación de los aspectos relacionados con la Psicología Clínica tradicional, actualmente se observa una marcada tendencia hacia criterios específicamente psicoeducativos. Sin embargo, las características de los centros privados limitan las posibilidades de elección de actuaciones por parte del profesional, pero, en general, se observa una tendencia hacia modelos actuales de intervención (programas, aspectos instruccionales, asesoramiento, etc.).

D) Se ha producido una gran proliferación de la oferta de gabinetes y centros de reeducación y apoyo escolar, con actuaciones de tratamiento muy diversas (logopedia, psicomotricidad, formación de padres, entrenamiento en habilidades básicas, etc.). Bastantes de estos centros se han consolidado y actualmente presentan una oferta de servicios de gran calidad y amplitud.

E) Es preciso señalar que en el campo de la educación especial se produce inicialmente cierta eclosión de ofertas de trabajo, al menos en los años ochenta, de asociaciones de padres de discapacitados psíquicos, sensoriales y motóricos; la progresiva cobertura por parte de las administraciones públicas de servicios en este sentido ha paralizado bastante esta vía.

\section{Las redes públicas de atención psicoeducativa}

La demanda social va aumentando en intensidad y llega a influir en las instancias político-administrativas, entonces en proceso de cambio en el contexto histórico de la transición democrática, planteando la necesidad de cubrir el espacio de intervención psicoeducativa por parte de las administraciones públicas. Fue en 1970, en la Ley General de Educación, cuando se formuló por primera vez el derecho a la orientación escolar, pero no es hasta 1977 cuando se crean los primeros servicios del M.E.C., los Servicios de Orientación Escolar y Vocacional (S.O.E.V.), con funciones (orientación personal, escolar y profesional, asesoramiento y apoyo al profesorado, información a padres, profesores y alumnos, investigación educativa, detección y diagnóstico de alumnos de Educación Especial) ligadas a los modelos de orientación de la época, muy amplias y poco definidas y con imposibilidad de efectuar una incidencia significativa en el sistema educativo, dado el escaso número de profesionales que inicialmente los componían (2-3 por provincia) y su compleja situación administrativa (situación que se hereda y aún se amplía en las Comunidades Autónomas con competencias educativas); se trata de profesores de Educación Primaria, psicólogos o pedagogos, al principio en "comisión de servicio" posteriormente como plaza de "carácter singular", sin reconocimiento del título superior exigido, lo que les conduce a una ardua batalla legal que todavía continúa. Tras un periodo de hibernación, las convocatorias de 1985 hasta 1989 nutren sus efectivos hasta 514 profesionales (253 psicólogos, 261 pedágogos) en el territorio de gestión del Ministerio de Educación (M.E.C.) en el momento de su desaparición legal (1992). Las CC.AA. realizan entre 1986 y 1990 múltiples convocatorias que duplican posiblemente el número mencionado en territorio M.E.C.

Paralelamente, a partir de las primeras elecciones democráticas municipales de abril de 1979, se crean Servicios Psicopedagógicos Municipales; en este caso lo que se produce es la adopción por los nuevos ayuntamientos de propuestas de profesionales (Psicología - Servicio Público) en las que se plantean formas de intervención del psicólogo desde el municipio que aún se sitúan en la indeferenciación educación/salud/servicios sociales, áreas que en ese momento no están cubiertas desde otras instancias. 
Los Servicios Psicopedagógicos Municipales (S.P.M.) estuvieron inicialmente muy influidos por los modelos comunitarios y preventivos procedentes del área de salud; y su distribución territorial (grandes urbes, áreas metropolitanas...) y condiciones de trabajo han sido muy variables y diversas; en España los ayuntamientos no tienen competencias directas sobre la red pública de enseñanza, aportan servicios complementarios por lo que la progresiva implantación de los servicios de intervención psicopedagógica de la administración educativa (inicialmente el M.E.C., progresivamente las comunidades autónomas) en los mismos sectores, limita la actuación de los S.P.M. a la oferta de programas concretos complementarios a la actividad escolar, muchos de ellos de gran calidad por cierto, todo lo cual paraliza su crecimiento.

Lo anteriormente dicho sirve exclusivamente para los S.P.M. de las grandes ciudades y sus áreas metropolitanas y no refleja lo ocurrido en Valencia (hasta el momento actual) y Cataluña (inicialmente) en que los procesos de colaboración de la autoridad educativa autonómica y los ayuntamientos adoptaron al menos temporalmente otras formas.

No disponemos de datos numéricos totales, pero vista la abundancia y diversidad de convocatorias y situaciones, podríamos especular con más de 1000 profesionales de la intervención psicoeducativa en todo el estado, trabajando en servicios municipales.

A partir del Plan Nacional de Educación Especial de 1977 y de la Ley de Integración Social del Minusválido de 1982, surge un nuevo marco de intervención psicoeducativa, se crean los Equipos Multiprofesionales (E.M., constituyéndose en Madrid en 1980), incrementándose en sucesivas convocatorias su número de forma importante en los años siguientes (hasta alcanzar en el momento de su desaparición legal los 796 profesionales, de ellos 231 psicólogos, el resto pedagogos, trabajadores sociales, logopedas y médicos); la situación de sus componentes era la de contratados laborales (con reconocimiento de su titulación) de la administración educativa tras concurso-oposición como forma de acceso. Sus funciones, originalmente ligadas a la educación especial, trataron fundamentalmente de dar respuesta a la necesaria evaluación inicial y toma de decisiones de ubicación de los alumnos susceptibles de recibir atención especial, por ello sus funciones se centran en: prevención, detección, valoración multiprofesional, orientación y seguimiento.

Estos Equipos incorporan perspectivas de trabajo interdisciplinar, normalización y sectorización (Gilolmo, 1989). Sin embargo se observa insuficiencia de recursos cuando las funciones asignadas son superadas por las demandas de intervención psicoeducativa de los sectores que atienden, también falta experiencia previa en el modelo planteado, y un enfoque inicial ligado a una concepción médicopedagógica de la educación especial hace difícil avanzar hacia actuaciones psicoeducativas más globales.

A partir del Real Decreto 334/85 de Ordenación de la Educación Especial (origen de las políticas de integración escolar) se abre un nuevo escenario. Por un lado se centran las funciones de los Equipos dependientes del M.E.C. en la valoración diagnóstica con vistas a determinar la ubicación escolar y en el apoyo a los proyectos y centros escolares de integración reconocidos a partir de dicha norma.

Además, se procede a la unificación de funciones de S.O.E.V. y E.M. (Orientaciones para el funcionamiento de Equipos Psicopedagógicos, enero 1986) planteando funciones generales en el sector, funciones en centros ordinarios y funciones específicas (Centros de Integración, Centros de Educación Especial....), un conjunto de prioridades (integración, atención a familias, diagnóstico, prevención y fracaso escolar) y un modelo de organización y funcionamiento (sectorización, plan de trabajo....).

Las líneas generales de estas orientaciones resaltan los aspectos de apoyo a alumnos y centros de integración, en general de atención individual y asesoramiento a centros, sólo en escasa medida menciona aspectos de "orientación" y no recoge más que muy parcialmente las aportaciones preventivas o sociocomunitarias tan abundantes en los documentos elaborados por los propios Equipos por la misma época; a partir de ese momento se produce una unifi- 
cación funcional, pero aún no de los aspectos administrativo-laborales, de los equipos ministeriales.

Con la puesta en marcha de la L.O.G.S.E. (M.E.C., 1990a) se va perfilando el modelo que la administración educativa plantea para la intervención psicopedagógica y la orientación educativa (M.E.C., 1990b), contemplándose dos niveles de actuación profesional; por un lado los Departamentos de Orientación en Educación Secundaria como apoyo técnico a la labor tutorial del profesor y recurso profesional de la orientación en el centro, con funciones referidas al propio centro, al alumnado, al profesorado, familias y otras instituciones; y por otro lado los Equipos de sector para facilitar a los centros de Educación Primaria una asistencia y apoyo técnico de naturaleza didáctica, psicológica, social y organizativa, además de funciones específicas de ámbito sectorial. También existen Equipos Específicos (Autismo, Sordos, Ciegos, Motóricos) de ámbito provincial y Equipos de Atención Temprana (que cubren la etapa de Educación Infantil).

Con el fin de cubrir las plazas de profesionales en los Departamentos de Orientación de los Institutos de Educación Secundaria y en los Equipos de Orientación Educativa y Psicopedagógica (E.O.E.P., nuevo y enésimo nombre de los Equipos de Sector del M.E.C.) se crea en 1991 la especialidad de Psicología y Pedagogía dentro del cuerpo de profesores de Educación Secundaria de carácter estatal (aunque la convocatoria de plazas en las Comunidades Autónomas con competencias educativas es prerrogativa de dichas comunidades) y se producen sucesivas convocatorias tanto centrales como autonómicas hasta desembocar en los más de 2000 miembros de dicha especialidad en 2002, de los cuales aproximadamente la mitad son psicólogos.

\section{Las competencias autonómicas}

El 1 de enero de 1982 se transfieren las competencias de gestión de la red educativa pública a las Comunidades Autónomas de: Cataluña, Galicia,
País Vasco, Comunidad Valenciana, Canarias, Andalucía y Navarra, el resto permanece como territorio de gestión del M.E.C. hasta el curso 1998/99 en que se produjo el traspaso de las competencias educativas al resto de las comunidades autónomas.

La mayoría de estas CC.AA. formulan modelos propios de organización de la intervención psicopedagógica, pero en general se mueven, al menos hasta 1991, hacia Equipos tipo S.O.E.V. (profesores de Primaria, psicólogos y pedagogos) en lo administrativo-laboral, y en la intervención señalan ámbitos comunes (atención a centros escolares, atención a necesidades educativas especiales, orientación y asesoramiento....).

A partir de la promulgación de la L.O.G.S.E. y sobre todo desde la creación de la especialidad de Psicología y Pedagogía en Secundaria, se han producido convocatorias propias para esa especialidad en la mayoría de las CC.AA. Y en general se tendía a organizar la actuación en Departamentos de Orientación en Secundaria y Equipos de sector para Infantil y Primaria si bien se han producido algunos modelos diferenciados: el caso señalado de la Comunidad Valenciana con interacción municipal y autonómica, el caso de Cataluña con EAPS en Primaria y Secundaria con discutibles coordinaciones con los Departamentos de orientación en I.E.S., el alarmante modelo gallego con un profesional por I.E.S, y hay suerte si sólo es un Instituto, más todos los Centros de Primaria e Infantil que de él dependen, más el resurgimiento de Departamentos de Orientación en Primaria dirigidos por un maestro, al que la titulación de Psicología o Pedagogía se le valora como mérito pero no se le exige, y últimamente el modelo navarro con desaparición de los Equipos de sector y adscripción de los profesionales a Centros de Primaria y Secundaria.

\section{Evolución de los modelos de intervención psicoeducativa}

El comienzo de los años ochenta marca el paso de una psicología educativa muy relacionada con 
modelos clínicos y de psicología "escolar" tradicional a una progresiva especialización y concreción en el objeto de trabajo. En esos años se produce, tardíamente, el descubrimiento de Vygotski y de la psicología cognitiva en relación con el contexto educativo (Filgueira y Esteban, 1991). Desde entonces el constructivismo se ha hecho dominante, esencialmente en el medio universitario relacionado con la Psicología de la Educación desde el que se ha extendido a la práctica profesional, de hecho ha llegado a ser el modelo psicopedagógico subyacente a las propuestas curriculares del proceso de reforma educativo formulado en la LOGSE.

La oficialización de dicho modelo, claramente no buscada por algunos de sus iniciales promotores, alcanzó niveles extremos y más allá de la teoría constructivista de base se introduce una perspectiva pedagógico-curricular, que, en nuestro caso, parte de propuestas para sacar la intervención psicopedagógica de la "periferia" del sistema educativo llevándola hacia el "núcleo" docente-didáctico-curricular (Maruny y Gómez-Granell, 1995). De esta idea se pasa a una reflexión de gran calidad pero curiosas consecuencias (propuesta de titulación de psicopedagogía, homologación de prácticas de psicólogos y pedagogos, priorización absoluta de los aspectos curriculares de la intervención.....) llegando hasta la asunción de estas reflexiones como posturas oficiales de la administración educativa y a desarrollos cristalizados y cerrados en los que se describe un modelo único, con unas únicas funciones, unas influencias teóricas concretas y cerradas, y se resumen el resto de las posibles funciones o formas de intervención como ajenas a la psicología educativa y propias de periclitados modelos psicométricos o clínicos (Martín y Solé, 1990; Monereo y Solé, 1996).

En conjunto se observa cómo la intervención psicopedagógica adopta un carácter "funcional" respecto a las necesidades de implantación de la reforma educativa, priorizando las actuaciones de asesoramiento curricular sobre las demás, formuladas, desarrolladas y realizadas con mejor o peor fortuna por los psicólogos educativos (Fernández Barroso, 1994).

\section{Líneas de actuación}

En este breve y muy incompleto recorrido queda patente que en la consolidación del rol del psicólogo educativo han confluido tres grandes líneas de actuación (Forns, 1994):

- Una, proveniente de un enfoque sociolaboral, que pretendía la inserción eficaz y madura del alumno en la vida activa (funciones de orientación profesional, asesoramiento vocacional).

- Otra línea de actuación es de tipo psicológico y pedagógico y pretende la mejora de la vida escolar, en una línea de apoyo a la comunidad educativa (funciones de asesoramiento, de formación de apoyo didáctico...).

- Una tercera línea puede ser caracterizada como de orientación terapéutica (reeducadora, compensadora...). Es la confluencia de estas lí-neas la que da al psicólogo educativo fuerza y riqueza conceptual.

\section{Presencia profesional y rol, dificultades y perspectivas}

En conjunto, los psicólogos educativos representan aproximadamente un $38 \%$ del total de los profesionales de la psicología que ejercen como tales, lo cual nos sitúa como una de las áreas más numerosas del colectivo profesional. En el sector público educativo se ha pasado en los últimos veinte años de la práctica inexistencia de psicólogos a los más de 3000 que pueden calcularse en la actualidad en las diversas administraciones municipales, autonómicas y centrales.

Las principales dificultades han venido por una doble vía, por un lado las relativas al estatus administrativo-laboral, en algunos casos por las formas de reclutamiento, en otros por problemas de reconocimiento profesional (afortunadamente cada vez en menor medida) y en bastantes, sobre todo en los que la intervención profesional es más reciente, por puros problemas laborales y socioeconómicos.

Otra fuente de dificultades, que además se hace más importante conforme se resuelven en mayor o 
menor grado los problemas de estatus, es la referida a la definición del rol, perfil y funciones; la progresiva superación de prácticas tradicionales y el surgimiento de la demanda social de intervención en distintos ámbitos produce la necesidad de un debate constante sobre las cuestiones referidas al rol del psicólogo educativo (Báez, 1993; Campos, F., 1995; C.O.P, 1994, Sanz y cols., 1991). A todo ello se unen unos desarrollos científicos y técnicos que obligan a un permanente esfuerzo de actualización y de definición de líneas nuevas de actuación psicoeducativa (como ocurre en general en todos los modelos y servicios educativos) que sin abandonar, como a veces se pretende, las grandes líneas de confluencia citadas en el apartado anterior, incorporen aspectos tales como los nuevos conceptos dinámicos del aprendizaje y de las formas de aprender, una evaluación formativa, analítica y basada en criterios, la respuesta a las necesidades derivadas de la integración de las diferencias tanto personales, como sociales y culturales, una orientación educativa y profesional de tipo educativo, el análisis del contexto educativo, etc. (Forns, 1994).

Finalmente se observa un tercer grupo de dificultades que van a ser clave para nuestro desarrollo profesional. Se trata de los aspectos deontológicos y éticos (Colodrón, 1998 y Fernández Barroso, 1997). Así nos encontramos los problemas de estatus y rol, la delicada frontera entre interdisciplinaridad e intrusismo, la dinámica entre funciones profesionales y funciones asignadas, la presencia de un rol mediador y una posición administrativa concreta y, finalmente, los dilemas éticos y deontológicos del psicólogo en contextos educativos: Entre estos últimos, cabe destacar las dificultades derivadas de los procesos de etiquetaje y consiguiente estigmatización, los temas de confidencialidad y secreto profesional, los requerimientos emanados del consentimiento para la intervención y, en última instancia, y como tema clave, la necesaria posición crítica del psicólogo ante el reforzamiento de un perfil institucionalmente deseable del alumno (França-Tarragó, 1996).

Por su trascendencia nos detendremos brevemente en este último punto. Se da un área de conflicto cuando el psicólogo educativo intenta crear, o apoya, la creación, en los alumnos, de determinados hábitos de pensamiento, sentimiento o conducta. Se trata de un punto en que la deontología profesional linda con la filosofía educativa. El análisis de sus distintas implicaciones trasciende el objetivo que nos proponemos en este texto. Sin embargo, es necesario tener en cuenta que el psicólogo puede dejarse utilizar por el "sistema institucional educativo" para inducir aquellos comportamientos o conductas que terminan beneficiando a los directores o educadores y no a los alumnos. Utilizar técnicas que promuevan la obediencia ciega, la sumisión, la competitividad y el individualismo supone una opción pedagógica y ética muy diferente a la de promover la autonomía y competencia personal, la creatividad, la cooperación, el respeto a los demás y la autodisciplina. El psicólogo educativo debe saber esto y tener en cuenta su propia posición en relación con los objetivos conductuales y actitudinales que se están intentando inculcar en los alumnos.

\section{Formación para la intervención profesional}

Es con respecto a las líneas de actuación antes citadas y a estos retos del presente y del futuro como podremos formular algunas reflexiones sobre la formación, tanto inicial como permanente, que requiere el psicólogo educativo para su desarrollo profesional. En primera instancia debemos tener en cuenta que un modelo formativo apropiado es aquel que avanza en una línea de profesionalización, entendiendo por profesionalización (Mauri, 1996) el proceso socializador por el cual los profesionales:

- Adquieren las características y capacidades específicas que les permiten ser competentes en su trabajo.

- Se mantienen en la dinámica del mercado laboral y se vinculan a un grupo profesional determinado.

- Se vinculan responsablemente a un contexto social específico, sobre la base de unos valores determinados. 
- Establecen las limitaciones propias de todo comportamiento experto, ya que ningún ámbito profesional posee todas las soluciones ni es todopoderoso.

Lejos de desvalorizar la práctica de los expertos, el reconocimiento de las limitaciones les conduce a reconocer y valorar el papel que otros profesionales tienen en el logro de los objetivos. Todos ellos conjuntamente, pero cada uno según lo específico de sus conocimientos, deben tratar de responder a las incógnitas que tienen planteadas, colaborando conjuntamente en ámbitos de coordinación que existen en las diferentes instituciones en que ejercen su trabajo.

El carácter polidimensional, contextual, ético, dinámico y cambiante de toda profesión contribuye a situar la formación en el núcleo mismo del ejercicio profesional y a que se la considere como uno de sus elementos característicos. Pero, debido justamente a lo que acabamos de establecer, no todos los enfoques posibles de formación son igualmente profesionalizadores. Desde este punto de vista, lo son únicamente aquellos que como profesionales nos ayudan a:

- Identificar, analizar y valorar críticamente los cambios sociales y culturales que es necesario llevar a cabo, puesto que inciden en la mejora de la sociedad.

- Reflexionar sobre el propio papel en la consecución de los mismos.

- Caracterizar la profesión integrando las exigencias que se derivan de los cambios previstos.

- Reforzar el compromiso ético que tenemos con aquellos a quienes nuestra actividad se dirige.

- Responder a las nuevas exigencias profesionales mediante el cambio real de la propia práctica profesional, elaborando respuestas a problemas nuevos y ejerciendo la autocrítica profesional.

En última instancia la formación ha de facilitar a los profesionales que construyan o transformen su saber teórico, conceptual, técnico-práctico y práctico. La formación debe integrar la reflexión en la práctica y sobre la práctica como un elemento clave de la preparación profesional, ya que todo ejercicio profesional se desarrolla en un contexto real, se vincula a ámbitos institucionales característicos del mismo y se organiza en función de actividades y situaciones prácticas familiares a todos los relacionados con la profesión.

\section{Algunas características de la formación del psi- cólogo educativo}

En la propuesta formativa adjunta al documento sobre el perfil profesional del psicólogo educativo elaborado por la Coordinadora Estatal del Colegio Oficial de Psicólogos-C.O.P. (C.O.P., 1998) se proponen cuatro niveles formativos. El primero de ellos incorporaría áreas propias del curriculum de la psicología, tales como:

- Psicología Evolutiva y del ciclo vital.

- Psicodiagnóstico aplicado a la educación.

- Personalidad, Psicopatología infantil y del adolescente.

- Psicología del Aprendizaje, Psicología Cognitiva.

- Orientación vocacional (escolar y profesional).

- Paradigmas, teorías y técnicas en Psicología de la Educación (conductismo, cognitivismo, constructivismo, sistémica...).

- Modelos de intervención en Educativa.

- Metodología Aplicada a la Psicología de la Educación.

- Psicología del lenguaje.

- Deontología Profesional aplicada al ámbito educativo.

Más algunas áreas complementarias, más específicas, como:

- Trastornos cognitivos y del lenguaje.

- Psicología Social aplicada a la educación.

- Psicología de las Organizaciones aplicada a la institución escolar.

- Intervención comunitaria.

- Conceptos básicos de neuropsicología aplicados a la educación.

Un segundo nivel se referiría al curriculum de otras materias y se referiría, sobre todo, al conocimiento del contexto educativo: 
- Contextos Educativos: Sistemas, estructura y organización.

- Didáctica.

- Nuevas tecnologías aplicadas a la educación.

El tercer nivel sería más práctico. Se trataría de la formación para el diseño y asesoramiento en programas referidos a las siete grandes áreas funcionales que se recogen en el citado documento sobre el perfil del psicólogo educativo (C.O.P., 1998). Estas son:

- Intervención ante las necesidades educativas de los alumnos.

- Orientación, asesoramiento profesional y vocacional.

- Prevención.

- Mejora del acto educativo.

- Formación y asesoramiento familiar.

- Intervención socio-educativa.

- Investigación y docencia.

Finalmente, el cuarto nivel sería la formación práctica en los aspectos relacionados con las áreas formativas antes expuestas, así como con la función propia del desempeño profesional del psicólogo educativo. En este nivel se incluirían las prácticas supervisadas, los procesos de tutorización por un profesional experto y, en general, la iniciación a la actividad profesional real.

\section{Una comparación}

Por primavera, al igual que brotan las flores y nacen los pajaritos, tiene lugar la convocatoria de oposiciones para Profesores de Enseñanza Secundaria (cada dos años en el agonizante territorio M.E.C., aleatoriamente o, al menos irregularmente, en las CC.AA.). Por la parte que nos toca de la especialidad de Psicología y Pedagogía, a este respecto podríamos realizar algunas reflexiones sobre lo que desde una perspectiva de intervención profesional podemos pensar sobre la formación y el reclutamiento del psicólogo educativo.

Resumiendo el punto anteriormente tratado, la perspectiva profesional concretada en los cuatro niveles citados combinaría la formación general en psicología educativa, el conocimiento del contexto educativo y de sus características, la concreción en programas de intervención y el inicio de la actuación profesional mediante una práctica supervisada.

Evidentemente, los procesos de reclutamiento y selección en cualquier profesión vienen determinados por la puesta en relación de las características de un puesto profesional con las de un candidato a través de un conjunto de técnicas y procedimientos; en este caso, el procedimiento es el concurso-oposición basado en un baremo y unas pruebas selectivas referentes a un temario. Realizando un somero análisis del temario de la especialidad de Psicología y Pedagogía podemos distinguir diversos bloques o agrupaciones de contenidos: Un primer bloque, digamos legislativo, trata de un grupo de temas de contenido "administrativo" - normas oficiales, leyes educativas y principios generales. Un segundo bloque trata del desarrollo de los alumnos en las distintas etapas y áreas. A continuación se plantean las materias transversales, se desarrolla la orientación educativa y profesional, seguidamente del bloque didáctico, pedagógico y de organización educativa, evaluación educativa, curricular y psicopedagógica, intervención educativa y adaptaciones en déficits específicos. Y, finalmente, un último bloque dedicado a la intervención en la comunidad educativa.

Aún teniendo en cuenta el ya citado, y muy discutible, carácter docente de la especialidad así como la indiferenciación de profesionales que a ella pueden acceder, el temario parece sobrecargado de conocimiento del contexto educativo (especialmente en la exposición de la compleja y prolija producción legislativa que ha generado el proceso de reforma educativa) y falto de temas específicos de la intervención profesional.

Sin embargo, más que en el contenido del temario, surgen problemas en la fase de concurso en la que la experiencia docente supone un paso decisivo en la obtención de la nota final (sin querer decir por ello que dicha experiencia no debiera ser valorable, sino que la experiencia profesional como psicólogo educativo debería ser valorada); incluso en el turno libre en que sería de esperar una menor importancia de este factor. 
Aunque en la fase final de la oposición se intenta que los candidatos hagan una aproximación práctica (a través de cuestiones y en la exposición del tema de "encerrona") se echa en falta alguna forma de evaluación de las capacidades de diseño y actuación en programas concretos referidos a la intervención.

Finalmente, y nos parece un aspecto clave, no existe una actividad equivalente al cuarto nivel que reflejábamos anteriormente, el nivel de práctica supervisada, una vez superados los procesos selectivos. Un primer momento de iniciación profesional junto a un psicólogo experto es una garantía de, al menos, un conocimiento de la intervención que muy dudosamente se adquiere sólo por un procedimiento de oposición.

\section{Conclusiones}

Si se me permite, reproduciré las conclusiones de mi más reciente trabajo (Fernández Barroso, 1998). De ellas se deducen, en mi opinión, unas consecuencias claves para los diseños formativos desde una perspectiva profesional.

El innegable crecimiento tanto numérico como en términos de estatus de los psicólogos educativos no puede, sin embargo, encubrir la persistencia e incluso el aumento de dificultades asociadas al rol profesional, acrecentadas por la participación, a veces incluso monopolización, de las entidades políticoadministrativas en la definición y priorización de funciones, sin excluir la necesidad de cierto grado de intervención administrativa, especialmente en los aspectos relacionados con la asignación de recursos, la homogeneidad y coherencia de las actuaciones y la gestión pública en general (Sánchez, 1991).

En este contexto, tan complejo como interesante, de crecimiento numérico y de estatus y de dificultades de rol, pensamos que como garantía de nuestra autonomía profesional y del servicio que nuestro trabajo presta, existe la necesidad de potenciar socialmente el conocimiento de nuestro rol y nuestras posibilidades de intervención.
Somos profesionales realmente existentes, con prácticas reales, mejores o peores, pero visibles y evaluables. Obtenemos avances y mejoras, intervenimos y somos reclamados por una creciente demanda social; los intentos de aprovechar una supuesta inseguridad asociada a problemas concretos - posición inestable de mediación, dificultades de formación y reclutamiento,.....- no pueden convertirnos en veletas, a merced de los vientos académico-universitarios o político-administrativos que en cada momento soplen.

En este mismo sentido creemos que es clave en la actuación de las organizaciones profesionales, C.O.P., Secciones, ,..., la oferta de actividades formativas que permitan el inicio y desarrollo profesional del psicólogo educativo, más en la línea de lo que en nuestro actual estado de conocimiento nos parece lo necesario para una intervención de calidad que lo que los citados vientos (que, no nos engañemos, responden a intereses concretos e inmediatos, ajenos a los, también, intereses pero, nuestros, de la profesión y de los profesionales) demandan.

La clave para la superación del actual estado de rol diluido es la realización por nuestra parte de una intervención de calidad, propia, abierta a la interdisciplinaridad pero específica, y en esta intervención la formación de calidad, tanto académica, como inicial y permanente, es quizás, el componente básico.

Somos psicólogos, somos los profesionales de la psicología cuyo objetivo de trabajo es el comportamiento humano en situaciones educativas y sólo mejorando nuestro desempeño, definiendo y perfilando nuestro rol, participando en las organizaciones e iniciativas profesionales, reflexionando y compartiendo nuestras reflexiones a través de las plataformas de expresión educativas y profesionales, avanzando en nuestra formación e investigando hipótesis de mejora, será como consigamos continuar nuestro desarrollo profesional y prestar un mejor servicio a la comunidad educativa y social en que nos desenvolvemos.

Finalmente, terminaremos citando a Jean-Claude Guillemard que al final del coloquio de Lille en 
1991 (A.F.P.S., 1992) señalaba que "son los propios psicólogos educativos y sus organizaciones los que tienen la obligación de dar a conocer a los que toman decisiones políticas que el desarrollo de los servicios de psicología educativa orientados hacia la transformación de la escuela es una baza positiva para los sistemas educativos del mundo entero".

\section{Referencias}

Báez, B. F. (1993). Elementos definitorios del rol del psicólogo escolar. Revista de Psicología General y Aplicada, 46, 465-473.

Campos, F. (1995). El rol del psicólogo de la educación. Papeles del Psicólogo, 63, 46-48.

C.O.P. (1998). Perfiles Profesionales del Psicólogo. Madrid: Colegio Oficial de Psicólogos.

C.O.P. Catalunya (1994). Proposta de redacció de l'estatut del professional de la psicología. Barcelona: C.O.P.-Catalunya.

Colodrón, M. F. (1998). Psicología educativa y deontología profesional. Papeles del Psicólogo, 71, 44-47.

Fernández Barroso, A. (Coord.) (1994). Rol, perfil y funciones del psicólogo educativo-Dossier. Madrid: C.O.P. Secretaría Estatal.

Fernández Barroso, A. (1997). Algunos aspectos deontológicos de la intervención profesional del psicólogo educativo de la red pública. En Jornadas Deontológicas. La Deontología: Garantía de calidad de los servicios psicológicos. Madrid: C.O.P.

Fernández Barroso, A. (1998). Desarrollo y situación actual de la intervención del psicólogo educativo en España. Papeles del Psicólogo, 71, 3-8.

Filgueira, M. y Esteban, A. (1991). La practique de la psychologie de l'education en Espagne. En A.F.P.S. Actes du Congrés de Lille. Lille: A.F.P.S. Forns, M. (1994). El psicólogo en el contexto edu- cativo. Anuario de Psicología, 63, 187-211.

França-Tarragó, O. (1996). Ética para psicólogos: Introducción a la Psicoética. Bilbao: Desclée de Brouwer.

Gilolmo, C. (1989). Psicología escolar. La evolución profesional en los últimos años. Papeles del psicólogo, 36/37, 90-95.

Guillemard, J. C. (1992). Mediation ou mediations psychologiques. En A.F.P.S. Actes du Congrés de Lille. Lille: A.F.P.S.

Maruny, L. y Gómez-Granell, C. (1995). La psicología de la educación en la España de los noventa. Psicología Educativa, 1, 7-24.

Mauri, T. (1996). La formación de los profesionales asesores: actualización y autoformación. En C. Monereo y I. Solé (Coords.) El asesoramiento psicopedagógico: una perspectiva profesional y constructivista (pp. 479-499). Madrid: Alianza.

Ministerio de Educación y Ciencia (1990a). Ley Orgánica de Ordenación General del Sistema Educativo. Madrid: Servicio de Publicaciones.

Ministerio de Educación y Ciencia (1990b). La orientación y la intervención psicopedagógica. Madrid: Servicio de Publicaciones.

Monereo, C. y Solé, I. (Coords.). El asesoramiento psicopedagógico: una perspectiva profesional y constructivista. Madrid: Alianza.

Muñoz, A. M., García, B. y Sánchez, A. (1997). La psicología en la escuela: Aportaciones a la historia de la psicología escolar. Psicología Educativa, 3, 7-26.

Sánchez, O. (1991). Los Equipos Interdisciplinares de Sector. Papeles del Psicólogo, 51, 40-45.

Sanz, L. M. y otros (1991). El rol del psicólogo educativo. Papeles del Psicólogo, 51, 51-52.

Solé, I. y Martín, E. (1991). Intervención psicopedagógica y actividad docente: Claves para una colaboración necesaria. En C. Coll, J. Palacios. A. Marchesi (Coords.). Desarrollo psicológico y educación (pp. 463-476). Madrid: Alianza Editorial.

Manuscrito recibido: 08/11/2010

Revisión recibida: 10/12/2010 Manuscrito aceptado: 03/01/2011 
Copyright 2011 by the Colegio Oficial de Psicólogos de Madrid ISSN: 1135-755X - DOI: 10.5093/ed2011v17n1a3 